\title{
Screening of plasmid-mediated MCR-1 colistin-resistance from bacteremia
}

\author{
P. Nordmann ${ }^{1,2,3}$ - L. Assouvie ${ }^{1,2}$ - G. Prod'Hom ${ }^{3}$ - L. Poirel ${ }^{1,2}$ • G. Greub ${ }^{3}$
}

To the Editor,

Recently, the very first plasmid-mediated colistin resistance (MCR-1) mechanism of resistance in Enterobacteriaceae was reported from animals, food and patients from China [1]. Then, it was reported worldwide, mostly in Escherichia coli. This finding is important since polymyxins are considered as last resort antibiotics for treating infections due to multidrug resistant bacteria.

Several pieces of evidence suggest that the reservoir of the mcr-1 gene might be related to animals, taking into account the heavy usage of polymyxins in veterinary medicine, and the fact that this gene was mostly identified from veterinary isolates worldwide. We have reported several human cases of infections due to $\mathrm{mcr}$-1-positive $E$. coli from community and hospital settings in Switzerland (Geneva and Neuchâtel regions) in 2015 and 2016 [2, 3]. When used in human medicine, polymyxins are mostly prescribed in hospital settings and the extent of diffusion of the $\mathrm{mcr}-1$ gene in human pathogens remain mostly unknown.

Therefore, a retrospective study was conducted to evaluate the spread of polymyxin resistance and MCR-1 positivity among enterobacterial isolates that had been isolated from clinically-significant specimens, i.e. blood cultures, at the

P. Nordmann

patrice.nordmann@unifr.ch

$1 \quad$ Emerging Antibiotic Resistance, Medical and Molecular Microbiology Unit, Department of Medicine, University of Fribourg, rue Albert Gockel 3, CH-1700 Fribourg, Switzerland

2 INSERM European Unit (LEA Paris), University of Fribourg, Fribourg, Switzerland

3 University of Lausanne and University Hospital Centre (CHUV), Lausanne, Switzerland
University Hospital of Lausanne (1,200 beds), Switzerland, in 2015. This study was performed in the same Frenchspeaking region of Switzerland as Geneva and Neuchâtel where $m c r-1$ positive $E$. coli isolates had been identified.

A total of 257 non-duplicated enterobacterial isolates was screened that included E. coli $(n=164)$, Klebsiella pneumoniae $(n=41)$, Enterobacter cloacae $(n=16)$, Klebsiella oxytoca $(n=14)$, Enterobacter aerogenes $(n=6)$, Citrobacter koseri $(n=5)$, Citrobacter freundii $(n=5)$, Hafnia alvei $(n=3)$ Kluyvera ascorbata $(n=1)$, and Salmonella serovar enteritidis $(n=2)$ isolates. The predominance of $E$. coli isolates in that collection is in accordance with the known enterobacterial distribution in bacteremia. The strains were screened first by using the recently-developed Rapid Polymyxin NP test that is more reliable and rapid to detect colistin resistance than disk diffusion, E-test and automated systems [4, 5]. Then, all strains were screened for the $m c r-1$ gene by using regular and real-time PCR techniques [3, 6]. Four isolates (three $H$. alvei and a single $E$. cloacae) were selected as resistant to colistin (prevalence rate, $1.17 \%$ ) according to the Rapid Polymyxin test. MIC determination confirmed that those four strains were indeed resistant to colistin (MIC $>2 \mathrm{mg} / \mathrm{L})$ [7], the E. cloacae strain showing an heteroresistance phenotype as known [8]. In fact, H. alvei might correspond to an enterobacterial subspecies being naturally resistant to polymyxins (P. Nordmann, personal communication). All the strains tested negative for the $\mathrm{mcr}-1$ gene.

This study indicated that the extent of diffusion of the $\mathrm{mcr}$ 1 gene is limited at least in this western part Europe. Very rarely reported in K. pneumoniae, the $m c r-1$ gene was accordingly not identified here in that species, which is an important nosocomial gram-negative pathogen. This finding may be good news for medical specialities that rely on broadspectrum antibiotics such as intensive care, heavy surgery and transplantation units. However, regular screening of 
plasmid-mediated colistin resistance is mandatory to detect the early occurrence of those polymyxin-resistant isolates and prevent their further spread.

\section{Compliance with ethical standards}

Funding This work has been funded by the University of Fribourg, and by the Office Fédéral de la Santé Publique, Switzerland.

Transparency declaration None to declare.

\section{References}

1. Liu YY, Wang Y, Walsh TR et al (2015) Emergence of plasmidmediated colistin resistance mechanism MCR-1 in animals and human beings in China: a microbiological and molecular biological study. Lancet Infect Dis 16:161-168
2. Nordmann P, Lienhard R, Kieffer N et al (2016) Plasmid-mediated colistin-resistant Escherichia coli in bacteremia in Switzerland. Clin Infect Dis 62:1322-1323

3. Poirel L, Kieffer N, Liassine N et al (2016) Plasmid-mediated carbapenem and colistin resistance in a clinical isolate of Escherichia coli. Lancet Infect Dis 16:281

4. Hindler JA, Humphries RM (2013) Colistin MIC variability by method for contemporary clinical isolates of multidrugresistant Gram-negative bacilli. J Clin Microbiol 51:16781684

5. Nordmann P, Jayol A, Poirel L (2016) Rapid detection of polymyxin resistance in Enterobacteriaceae. Emerg Infect Dis 22:1038-1043

6. Bontron S, Poirel L, Nordmann P (2016) Real-time PCR for detection of plasmid-mediated polymyxin resistance ( $m c r-1)$ from cultured bacteria and stools. J Antimicrob Chemother. 2016 Apr 27 [Epub ahead of print]

7. European Committee on Antimicrobial Susceptibility Testing (EUCAST) (2014) Breakpoints tables for interpretation of MICs and zone diameters, Version 2.0. EUCAST

8. Band VI, Crispell EK, Napier BA et al (2016) Antibiotic failure mediated by a resistant subpopulation in Enterobacter cloacae. Nat Microbiol 09:16053 